\title{
Blur Image Classification using Object Focusing Technique in E-Governance
}

\author{
Sandeep Kumar \\ Research Scholar, Bhagwant \\ University Ajmer
}

\author{
Rajeev Kumar, PhD \\ College of Computing Sciences \\ \& IT, Teerthanker Mahaveer \\ University Moradabad
}

\author{
Meenu Kansal \\ Research Scholar From Mewar \\ University (India)
}

\begin{abstract}
usually whenever Images is captured by satellite in different environment Like Landscape, forests, Hills, Dark, Shiny, Oceans Region and Different Geography. Than we have to improve these images because without clear images it's really hard to get information. There are several methods used to improve the observation of these images like Histogram Equalization Technique, Local Histogram equalization technique, Discrete Cosine Transform, and Discrete Wavelet Transform method(DWT). All these technologies face troubles like failure of image info, loss of edge details etc. Wavelet transforms have become one of the very important and very powerful tool of signal representation and we can enhance our images by using this technique. Bicubic interpolation is used as an midway stage for appraising high frequency components and it is more refined than the nearest neighbor and bilinear techniques. The proposed technique has the benefits of superior resolution, sharper image and smoother edges by the DWT and bicubic.
\end{abstract}

\section{Keywords}

Image Enhancement, DWT, Bicubic interpolation, satellite catpture image.

\section{INTRODUCTION}

Image enhancement is a technique to increase the image/picture quality for the human observer by the different technique. The processing helps in max clearness, sharpness and details of features of interest towards information extraction and further study. One of the most essential quality factors in images comes from its resolution. Interpolation in image processing is a well-known method to rise the resolution of a digital image. Interpolation has been usually used in many image-processing techniques for example facial reconstruction1, resolution enhancement and multipledescription coding.

For increasing the image resolution we have different type of interpolation technique. Usually Interpolation techniques are based bilinear, bicubic, Lanczos and on nearest neighbors. By the Bilinear Interpolator we get accurate interpolating image with the nearest neighbor method but also requires more processing time.

Wavelets are also playing a major role in many image processing applications. Which is mostly divided into discrete wavelet, continuous wavelet and multi resolution based transforms. Wavelet coefficients can be assessed in order to increase sharpness of the image.

The DWT decomposes input image into four sub bands
a) LL band b) LH band c) HL band d) HH band

The frequency components of those sub bands cover the full frequency spectrum of the original image.

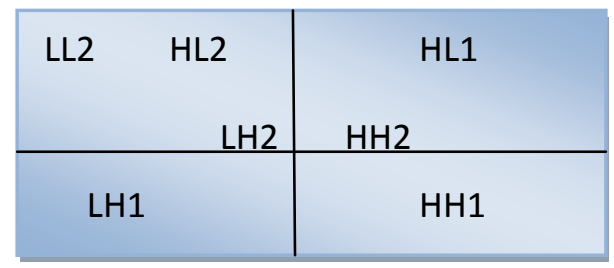

Figure 1: The 2D wavelet filter bank

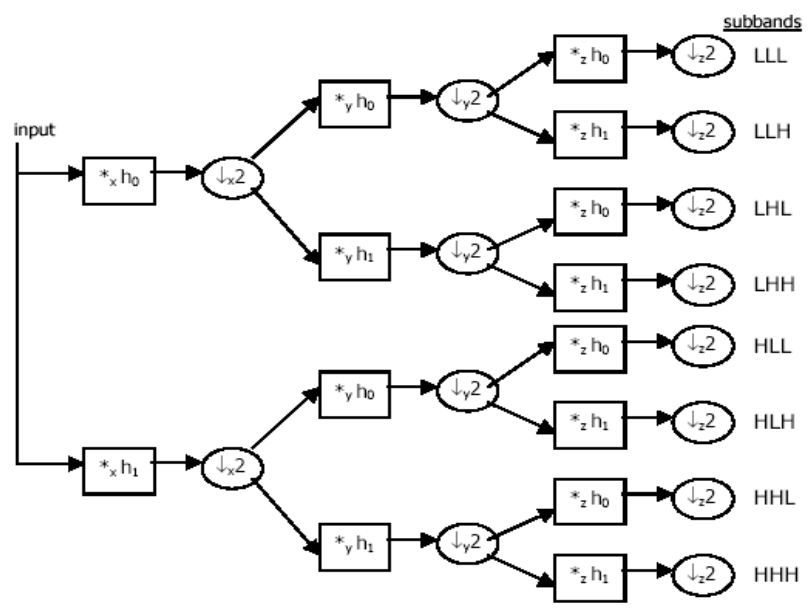

Figure 2.0 2D Filter Bank diagram

\section{LITERATURE SURVEY}

There is number of methods are used for improving the quality of images in many applications. Every technique through different objects and outcomes. Hasan Demirel and Gholamreza Anbarjafari [11] proposed a DWT technique for interpolating the images. But the images obtained from IDWT and DWT method is not so clear and enhanced, it has low PSNR as compared to other methods. Hasan and Gholamreza [12] covered the Discrete and stationary wavelet decomposition method based on interpolation of high frequency sub-band images resulting from DWT. In this method high frequency components of image are improved by stationary wavelet transform. This method produces quite better results. Hasan Demirel and Gholamreza Anbarjafari [13][15] discussed Complex Wavelet Transform (CWT) which is used in image processing. CWT yields two complexvalued low-frequency sub-band images and six complex valued high frequency sub-band images of original image. MSE and PSNR of the super resolved image is also develops in this method. By analyzing correlation between lower level 
and higher level sub-bands, interpolation filters are designed. Initially filters are estimated by smearing wavelet transform to low resolution image. Estimated filters are then used to estimate bands in advanced level. Finally, inverse wavelet transform is achieved to increase the resolution of input image

\section{ENHANCEMENT OF SATELLITE IMAGE RESOLUTION}

A common feature of wavelet domain image resolution enhancement algorithm is the theory that LR image to improve is the LP filtered sub-band of high resolution image which has been exposed to decimated wavelet transform2

\subsection{Wavelet Domain Zero Padding (WZP)}

In WZP an initial estimation the unknown HR image is generated using wavelet-domain zero padding (WZP). This calculation commonly shows objects such as smoothing and ringing .Using LR image $\mathrm{x}$ of size $\mathrm{m} \mathrm{x} n$, the anonymous $\mathrm{HR}$ image $y$ is recreated by using zero padding of high-frequency sub-bands (i.e. setting all components of these sub-bands to zeros) followed by inverse wavelet transform:

$$
\hat{\mathbf{y}}_{0}=W^{-1}\left[\begin{array}{cc}
\mathbf{x} & \mathbf{0}_{\mathbf{m}, \mathbf{n}} \\
\mathbf{0}_{\mathbf{m}, \mathbf{n}} & \mathbf{0}_{\mathbf{m}, \mathbf{n}}
\end{array}\right]
$$

such as $0 \mathrm{~m}, \mathrm{n}$ is an all-zero sub is an all-zero submatrix of dimensions $\mathrm{m}^{*} \mathrm{n}$ and $\mathrm{W}-1$ is the inverse discrete wavelet transform3.

\subsection{Cycle Spinning and Edge Modeling}

The ( DWT) decimated wavelet transform is not shiftinvariant and as an outcome, distortion of wavelet coefficients, due to quantization of coefficients in compression applications or non-exact estimation of high frequency coefficients in resolution enrichment applications (including zero padding of coefficients as in WZP), introduces cyclostationarity into the picture/image which manifests itself as ringing in the neighborhood of gaps.

Cycle-spinning (CS) has been shown to be a good effective manner against ringing when used for de-noising purposes in the wavelet domain [5][16] and also for decreasing ringing and increasing the perceptual worth of compressed images. CS method aims to estimated shift-invariant data by averaging out the cyclostationarity. In [6][17] and [8], it was shown that Cycle Spinning (CS) applied as a post-processing operation after decompression results in major enhancements in the framework of JPEG and JPEG2000 image compression.

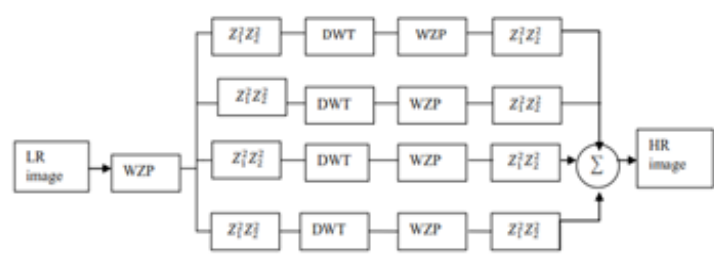

Figure 3. WZP and CS based image resolution

Edge detection is the method used most recurrently for segmenting images based on abrupt changes in intensity. We begin by introducing several ways to model edge and then discuss a number of their intensity summaries. a steps edge involves a transition between two intensity levels arising ideally over the distance of 1 pixel.
Digital images have edges that are blurred and noisy, with the degree of blurring determined principally by constraints' in the focusing mechanism, and noise level determined principally by electronic component of imaging system. In such situations, edge is more closely modeled as having an intensity ramp profile. An edge segment would then be a set of such points that are connected.4

\subsection{Image Resolution Enhancement Using Inter-Sub-band Correlation in Wavelet Domain}

When a image capture by satellite than some time image has low resolution, the image will be not clear that is look like blur image. Hera the frequency is very low and discontinuous, so the property is shift variant due to frequency is discontinuous, the property keeps on changing. If the resolution is high, the image will be clear because the frequency will be as much as continuous. The main advantage of using Inter-Sub band correlation is we can get continuous frequency, so the shifting property keeps invariant. The low resolution image will get clear and enhanced.

Phase-shifting matrix was first introduced by Xin $\mathrm{Li}[9][14]$ for saving the computational difficulty of LBS [10] method. It showed that two sub-bands of a two-channel perfect restoration filter bank in Fig. were linked by a unique phaseshifting matrix.

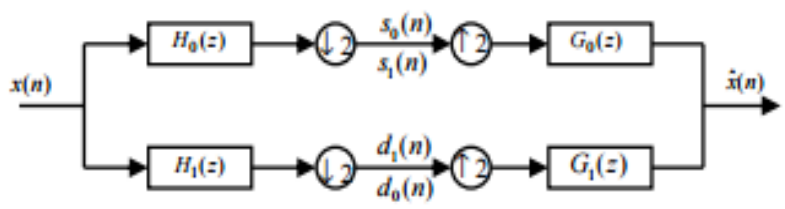

Figure 3.2:- two channel filter bank

\section{PORPOSED METHOD}

\subsection{Dwt-Based Resolution Enhancement}

In this paper, DWT is used to save the high-frequency modules of the image.

Wavelets play a main role in numerous image processing applications. The 2-D wavelet decomposition of an image is achieved by relating the 1-D DWT along the rows of the image primary, and thenthe fallouts are decayed along the columns.

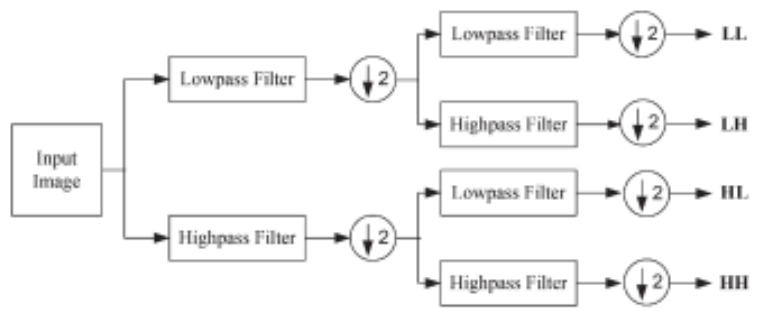

As the above figure shown DWT separates the image into different subband images. First by using horizontal filters to obtain LowerPass and HighPass Filter, and then by using vertical filters LL, $\mathrm{LH}, \mathrm{HL}$, and $\mathrm{HH}$. 


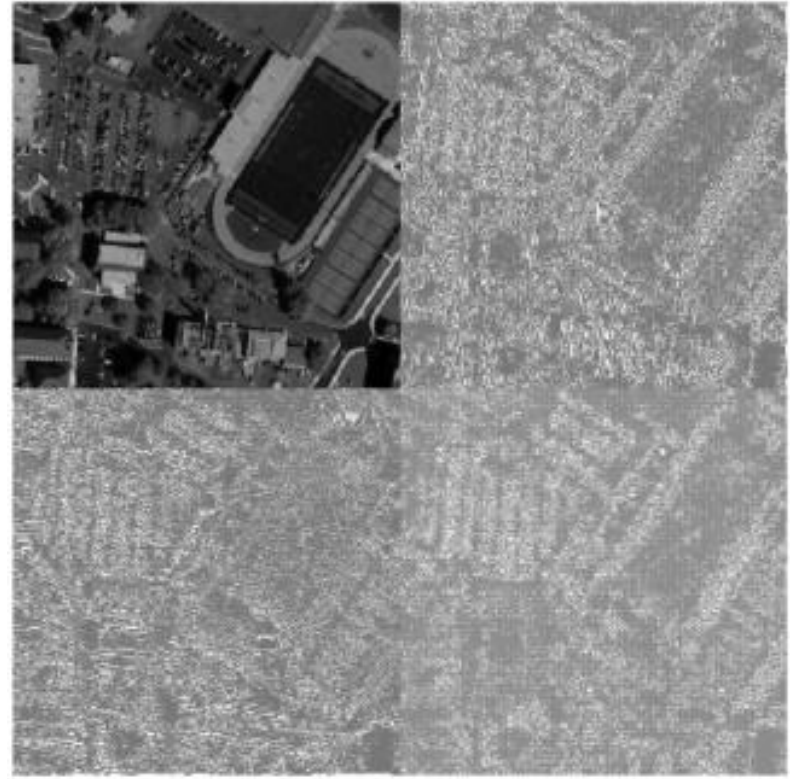

Fig:- 4.1 LL, LH, HL, and HH subbands of a satellite imageobtained by using DWT

\subsection{Bicubic interpolation}

Is approved out to all the quadrants distinctly after DWT interpolation of image. Bicubic interpolation (BI) is the default pixel interpolation algorithm and it produces each target pixel by interpolating the nearest sixteen mapped source pixels. The interpolation objects like blurring and aliasing can be greatly reduced by bicubic interpolation.7

\subsection{Algorithm}

1. For the Analysis capture a low contrast satellite image as input.

2. Use general histogram equalization method for Equalize.

3. After the equalization, compute the discrete wavelet transform (DWT) for the contrast enhancement.

4. DWT of an image decomposed four sub-band images referred to as (LL, LH, HL, and $\mathrm{HH}$ ).

5. The intermediate process of adding the difference image, containing high-frequency components,

6. Get output Image.

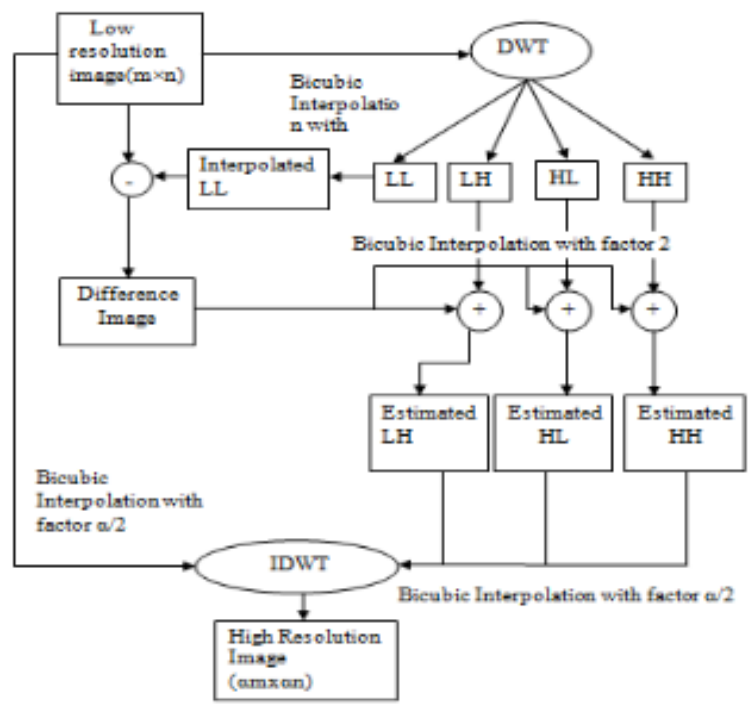

Not only graphical comparison but also quantitative comparisons is endorsing the supremacy of the proposed

method. Peak signal-to-noise ratio (PSNR) and root mean square error (RMSE) have been implemented in

direction to obtain some computable results for evaluation. PSNR can be obtained by using the following formula:

PSNR $=10 \log _{10}(\mathrm{r} 2 / \mathrm{MSE}) \ldots \ldots 1$

Where $\mathrm{R}$ is the max. fluctuation in the input image (255 in here as the images are denoted by 8 bits) and

MSE is expressive the MSE between the assumed input image I

$$
\begin{aligned}
& \text { acquired by the following } \\
& M S E=\frac{\sum_{i, j}\left(I_{\text {in }}(i, j)-I_{\text {arg }}(i, j)\right)^{2}}{M \times N}
\end{aligned}
$$

Where $\mathrm{M}$ and $\mathrm{N}$ are the size of the images.

Obviously, RMSE is the square root of the MSE, hence it can be deliberate by the following:

$$
R M S E=\sqrt{\frac{\sum_{i, j}\left(I_{\text {in }}(i, j)-I_{\text {arg }}(i, j)\right)^{2}}{M \times N}}
$$




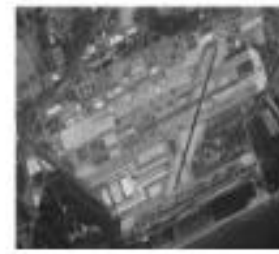

(a)

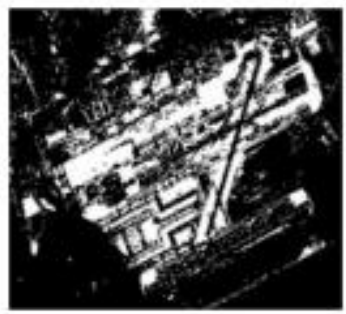

(c)

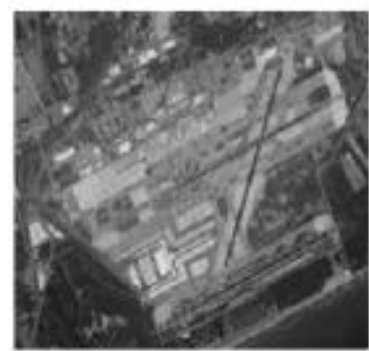

(e)

Figure 3.3 Satellite Image of US-Topo (a) Low Resolution Input Image, Resolution Enhanced Images using (b) Bilinear Interpolation, (c) Bicubic Interpolation, (d) Wavelet Zero Padding, (e) DWT based Image decomposition and bicubic interpolated image

\section{PSNR and RMSE}

WZP Bilinear Bicubic $\square$ DWT \&Bicubic

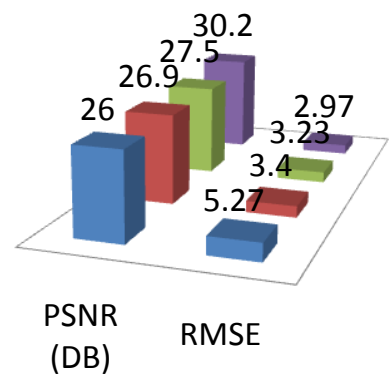

The proposed approach is higher by the PSNR of DWT and bicubic interpolation technique.

PSNR (Decibels) results for resolution enhancement for the proposed technique compared with conventional and some state-of-art techniques shown in chart

\section{CONCLUSION \& FUTURE WORK}

In this Paper we achieve more enhanced and clear image by proposed technique. Where Both the tested RMSE , PSNR and visual Results Shows that we get a best result by the proposed technique over the traditional and conventional and state-of-art image resolution enhancement techniques

For future work of the project we try color image development instead of gray scale image Enhancement. Whichcan improves the efficiency of the input Sample images by removal of noise. Also it may include the enhancement of satellite image with moving objects.

\section{REFERENCES}

[1] Y.-B. Li, H. Xiao, and S.-Y. Zhang, "The wrinkle generation method for facial reconstruction based on extraction of partition wrinkle line features and fractal interpolation," in Proc. 4th ICIG, Aug. 22-24, 2007, pp. 933-937.

[2] Mohan, K.B. Jayarraman, U. Maheswaran, D. Sathiyaraj. G.Dhakshanamoorthi"A Novel Approach for Satellite Image Resolution Enhancement" International Journal of Engineering and Advanced Technology (IJEAT) ISSN: 2249 - 8958, Volume-2, Issue-4, April 2013.

[3] AlptekinTemizel, Theo Vlachos,"WAVELET DOMAIN IMAGE RESOLUTION ENHANCEMENT USING CYCLE SPINNING AND EDGE MODELLING" in Eusipco2005.

[4] Rafael C Gonzalez,”Digital image processing ”.

[5] Temizel and T. Vlachos, "Wavelet domain image resolution enhancement u sing cycle-spinning," Electron. Lett., vol. 41, no. 3, pp. 119-121, Feb. 3, 2005.

[6] A. Nosratinia, Denoising of JPEG Images by Reapplication of JPEG”, Journal of VLSI Signal Processing, vol. 27, no. 1, nal of VLSI Signal Processing, vol. 27 , no. 1

[7] Remimol.A.M "A Method of DWT with Bicubic Interpolation for Image Scaling" Remimol.A.M et al. International Journal of Computer Science Engineering (IJCSE).

[8] A. Nosratinia, "Post-Processing of JPEG-2000 Images to Re move Compression Artifacts", IEEE Signal Proc. Letters, Vol. 10, No. 10, pp. 296-299, Oct. 2003.

[9] Xin Li, "New Results of Phase Shifting in the Wavelet Space", IEEE Signal Processing Letters, Vol.10, No.7, July 2003.

[10] H.W. Park, H.S. Kim, "Motion Estimation Using LowBand-Shift Method for Wavelet-Based MovidngPicture Coding", IEEE Trans. On Image Processing, vol 9, No.4, pp 577-587, April 2000.

[11] Hasan Demirel and Gholamreza Anbarjafari, 2011, "Discrete Wavelet Transform-Based Satellite Image Resolution Enhancement”, IEEE Trans. ON Geoscience and Remote Sensing, vol. 49, no. 6.

[12] Hasan Demirel and Gholamreza Anbarjafari, 2011, "Image Resolution Enhancement by Using Discrete and Stationary Wavelet Decomposition", IEEE Trans. on Image Processing, vol. 20, no. 5.

[13] R.Vani1, Dr. R. Soundararajan, 2013, "DWT and P C a Based Image Enhancement with local Neighborhood 
filter Mask", IOSR Journal of Computer Engineering, 8727Volume 9, Issue 2, PP 67-70

[14] Rajeev Kumar, M.K. Sharma, “Advanced Neuro-Fuzzy Approach for Social Media Mining Methods using Cloud", IJCA: International Journal of Computer Application; Volume 137 - No.10, March 2016: 56-58.

[15] Rajeev Kumar, Dr. M.K. Sharma, "Collision of ICT for Cloud Computing in e-Governance". New York Science Journal 2013; 6(5):78-80.
[16] Rajeev Kumar, Dr. M. K. Sharma, "Cloud Application of e-Governance System Using Advanced Wireless Networks". International Journal of Researcher 2013; $5(6): 26-29$

[17] Rajeev Kumar, Dr. M.K. Sharma, "Advanced Architecture Algorithm of Sensor Based Robotics Security System Framework for e-Governance Technology", International Journal of Computer Application, April 2012. Volume 43, Issue 3, pp- 1-4. 